le portiQue Le Portique

le portiQue Revue de philosophie et de sciences humaines

$10 \mid 2002$

Les paradis artificiels

\title{
Addictions : à corps perdu
}

Dominique Marinelli

\section{(2) OpenEdition}

Journals

Édition électronique

URL : http://journals.openedition.org/leportique/140

DOI : 10.4000/leportique.140

ISSN : $1777-5280$

Éditeur

Association "Les Amis du Portique"

Édition imprimée

Date de publication : 1 septembre 2002

ISSN : 1283-8594

Référence électronique

Dominique Marinelli, « Addictions : à corps perdu », Le Portique [En ligne], 10 | 2002, mis en ligne le 06 juin 2005, consulté le 08 avril 2021. URL : http://journals.openedition.org/leportique/140 ; DOI : https://doi.org/10.4000/leportique.140

Ce document a été généré automatiquement le 8 avril 2021.

Tous droits réservés 


\section{Addictions : à corps perdu}

\section{Dominique Marinelli}

À mes patients et à quelques autres

1 La turbulence des drogues et des paradis artificiels montre des pathologies sous la forme de dérives de l'oralité. Elles concernent la toxicomanie, l'anorexie, la boulimie, l'alcoolisme, le tabagisme, le jeu, etc. Les discours officiels les regroupent actuellement sous la terminologie de dépendances ou d'addictions ${ }^{1}$. Ce terme issu de l'anglais signifie "l'attachement, l'inclinaison, le fait de s'adonner à quelque chose, à quelqu'un. Il correspond $\grave{a}$ une urgence $d u$ besoin et $\grave{a}$ l'insuffisance finale de toute tentative de satisfaction $»^{2}$. Si les conséquences de leurs abus sont souvent graves, ces dérives sont reconnues être vécues, avec plus ou moins de prégnance, par tout un chacun qui mange, qui boit... qui vit et qui parfois défaille. La fragilité de notre tension orale est évidente et la bascule dans l'excès est vite inaugurée. Insidieusement, l'alcool, le tabac, la nourriture, la drogue s'affranchissent de tout contrôle et exigent leur première place dans l'économie désirante de l'un ou de l'autre.

Dans ces affections de l'oralité, quelque chose est repérable d'emblée, quelque chose d'imparable. C'est le corps et l'usage, pourrait-on dire, qu'en fait celui ou celle qui est touché par cet affront atteint par cette plaie. Touché bien sûr, mais de façon violente, atteint de plein fouet, comme on l'est d'une balle percutant sa cible. Traversé, transpercé, comme si ce corps n'était pas à sa place, pas en place, tenant si mal debout, décalé. L'anorexique veut aplatir son corps le plus possible et y perdre sa ligne de vie. Le boulimique le remplit jusqu'à plus soif, il avale, il engouffre jusqu'à vouloir le faire éclater, peut-être. L'alcoolique s'en va de guingois, il a perdu son balancier. Le toxicomane le transperce, le pique, le troue..., pour y faire entrer la liqueur magnifique. Le joueur use de sa nonchalance, le souffle coupé et la peur au ventre, «Like any dealer he was watching for the card that is so high and wild, he'll never need to deal another " ${ }^{3}$, etc. Une intense fébrilité secoue ces corps.

3 Ces oralités, à la dérive, griffent le corps, afin de tenter d'en (re)trouver quelque trace, d'en définir la précision d'un chemin, fait de vicissitudes (?). Elles essaient de départager le corps, de le couper, d'en repérer une ligne médiane, qui suivrait le tracé de l'œsophage, lieu où passe ce qui est avalé, la bouche, lieu où sont ingérées toutes 
sortes de choses, les veines où passe ce qui est injecté. Les sensations recherchées et trouvées par l'ingestion ou l'injection des produits, ou leur rejet pour l'anorexique, font que cela irradie, se propage, se répand, tout le corps s'en ressent, les spasmes en secouent l'ensemble. L'oralité se révèle parce qu'elle emprunte cette ligne à peu près médiane qui permet de faire tenir le corps. Pour l'anorexique, le boulimique, l'alcoolique, la sphère orale est privilégiée. Ce sont souvent les veines pour le toxicomane. Et le corps entier, chez les mystiques serrant «leur haire avec leur discipline $»^{4}$.

4 L'utilisation du terme ancien d'addiction date du Moyen Âge. Il signifiait une contrainte par corps infligée à des débiteurs ne parvenant pas à régler autrement leur créanciers. Donner son corps pour une dette impayée. Retrouver le chemin du corps par le corps, retrouver le chemin du corps en lui passant au travers.

Dans le miroir, « l'addicté $~^{5}$ voit une image biaisée, une image extensible, une autre image que sa réalité, comme un mirage, mais aucune oasis rafraîchissante, salvatrice, ne s'y profile. Un regard s'est perdu. L'image est au-delà de la réalité, elle est figée et manque de relief, elle est sans perspective, sans arrière-plan, rien ne la soutient, elle se gondole, se brouille. C'est l'image d'un corps aux limites floues. Le regard ne peut la soutenir, il est fuyant, il croit embrasser le paysage à perte de vue, mais ne retient que le vide intolérable. Le toxicomane, l'alcoolique regardent ailleurs, leur regard, peutêtre, se porte sur le corps semblable du voisin, y espérant une réplique.

6 Cette image est faussée, le corps tangue et chavire, chamboulé, d'où ce besoin forcené, inéluctable d'ingurgiter ou de dégurgiter, de piquer, de marquer ce corps souvent par le piercing, ou les tatouages, de faire passer quelque chose par la bouche ou par tout autre partie de corps puisqu'elles sont équivalentes. Ce besoin de tracer un chemin à suivre pour que s'inscrive une bonne fois la satisfaction de cette sensation recherchée. En vain. Faire passer sur le corps, à l'intérieur du corps, recréer un circuit sensitif, pulsionnel pour se sentir exister, pour retrouver, pour récupérer un corps par ces sensations fortes. Pour recréer une sensation première? Un apaisement, une satiété fournis par la nourriture, l'alcool, la drogue... ? Un plaisir retrouvé, assouvi, croit-on.

7 Mais ce trajet des retrouvailles est faussé et la répétition dans sa fixation au « toujours même " s'installe. Il faut sans cesse le remettre en acte, pour rejouer une perte, pour tenter d'extraire cette sensation incorporée de travers.

8 Cela se fixe car la distance ne se marque pas dans le corps à corps de son propre corps avec son image. Cent fois «l'addicté » remet son ouvrage corporel sur le métier des excitations qui n'ont pu être élaborées, faute de mots, sans doute, la problématique étant orale.

9 Des mots ne sont pas venus définir son corps, le délimiter. Pas de mots médians, pas de paroles vraies, sécurisantes, médiatrices, rien que le vide. Si la médiatisation par une parole apaisante, consolatrice, délimitant, parant les excitations, n'a pu se faire, "l'addicté » est soumis à une ré-insistance de son besoin, à un dérapage incontrôlé dans ce circuit fermé : de la virulence du besoin à sa satisfaction immédiate. En effet l'excitation est forte, de plus en plus forte, quelque chose passe par la bouche, et va vers l'intérieur du corps; cela a comme premier effet un certain apaisement, mais si l'excitation se reproduit, si des paroles pouvant aider à différer ce besoin, sont absentes, elle devient trop forte, trop prégnante, elle ne laisse aucun espace au manque, à un effet de symbolisation, d'où la fermeture de ce circuit. Il n'y a aucune possibilité de jeu, de remplacement par autre chose, comme le fait le nourrisson avec le 
suçotement par exemple. Pas de parole "différante " ${ }^{6}$. Le nourrisson investit son pouce quand il est en peine de se satisfaire oralement, il n'a pas toujours besoin de nourriture, sa mère n'est pas toujours immuablement présente, et heureusement ! C'est pour lui une tentative de réponse à un manque réel ${ }^{7}$, une réponse à une privation. Il trouve, il sait remplacer quand quelque chose manque à l'appel de la pulsion excitatrice, quand quelque chose laisse à désirer. Mais si chaque fois l'illusion est donnée d'un assouvissement rapide, par le lait chaud, par exemple, sans paroles accompagnatrices, sans mots d'amour, le nourrisson ne pourra symboliquement remplacer cette quête de satisfaction. L'espace se clôturera d'emblée par la monotonie de la répétition sans créer un espace imaginaire pouvant amender le manque et mettre du jeu, aérer la confusion des sensations.

10 La perception sensitive, sensible, reste ainsi à l'état brut, l'excitation trop déroutante, douloureuse, est sans cesse réactivée, aucun écart n'est possible, la part imaginative est absente. La quête est d'une avidité mortifère car la réponse n'est que réponse au corps à corps. C'est un accrochage corporel qui s'installe à défaut d'un accrochage imaginaire, la symbolisation étant en panne: pas de mots pour signifier le manque, pas de mots pour dire l'angoisse, pas de mots pour que se ferme la plaie sur le corps. Une réponse réelle, une trace sur le corps, marquée par la souffrance. Dans ce cas, il n'y a aucun moyen d'habiter le vide, de l'apprivoiser, en laissant place à l'imaginaire. La dépendance ne peut que s'installer, à un autre corps d'abord, à des produits de substitution ensuite.

11 La réponse par l'incorporation de produits va tenter de faire la nique à cette angoisse, mais, c'est un leurre, de se faire croire qu'en planant le monde sera plus beau, plus supportable, qu'en buvant, le courage reviendra, qu'en se remplissant de nourriture, l'angoisse ira faire son trou ailleurs, que l'on pourra remplir le vide qui s'agrandit, qui prend toute la place, qui vrille le corps. La confrontation à ce vide est intolérable. L'angoisse les submergeant, la fébrilité les assaillant, le besoin se fait sentir de plus en plus de devoir colmater cette brèche à tout prix, de remettre en route le circuit, de plus de substances, plus de maîtrise, plus de jeûne, plus de traces pour sentir son corps être là, un peu. À partir d'un moment, cependant, les sensations s'estompent, le corps se fait brumeux, l'esprit aussi.

12 Ce corps est un corps déshabité, un corps qui n'est pas investi comme le sien propre. Il est un élément extérieur, regardé d'en dehors de soi, au lointain de soi. C'est un corps qui n'est pas à la place d'un corps existant pour quelqu'un, un corps qui est dans l'incapacité de fonctionner tout seul, parce que justement il est dans l'incapacité de se mêler aux autres corps, de se vivre en relation avec les autres corps, parce qu'il ne saurait s'en différencier. Seul, il n'a pas droit de cité, il est pris par un autre corps, il est sous dépendance, sous influence, en lien éternel. «L'addicté » est sans cesse accroché à quelqu'un qui le traîne, à une histoire qu'il redit sans fin, qu'il se répète en boucle, ne pouvant s'y faire croire.

13 Le corps réclame toujours plus de nourriture, de substances, de toxique, de médicaments, etc., de plus en plus de rien pour l'anorexique. Mais quand le toxique prend toute la place, tout le temps, la maigreur arrive aussi, pour le toxicomane, pour l'alcoolique, le corps se délite petit à petit. Besoin de se remplir à en vomir, de se vider à en mourir, de se piquer pour se suivre à la trace, pour remplir les petits trous, ne pas laisser de blanc et suivre le parcours fléché d'un corps en suspension. Ou sentir autour de ses lèvres, ou manipuler entre ses doigts la cigarette salvatrice, réconciliatrice, 
régénératrice. La caresser des lèvres ou des doigts, la tourner, la retourner, ou téter une pipe ou un cigare, toujours à la recherche de sensations plus que vraies?

Une vie ritualisée se montre ainsi dans la consommation accrue "d'objets", mais ce rituel se joue dans l'accession à ces produits, dans le tout faire pour pratiquer cette cérémonie "immuable de la possession de l'objet ${ }^{8}$ et tout faire pour déjouer les empêchements de jouir en rond, n'avoir en perspective que cette recherche qui fait que plus rien d'autre n'existe, plus rien d'autre ne vit.

Quels liens tente-t-on de recréer ainsi, de faire subsister pour faire tenir ce corps qui s'étiole, qui s'effiloche? Pourquoi devoir en passer par "l'épreuve du corps " ${ }^{9}$ ? Le corps vécu, pris comme un tas de chair en souffrance où tout fait mal, tout se bouscule, se cogne, tout est confus, sans queue ni tête (?), est un corps qui ne se vit pas, qui ne peut se vivre comme manquant. "L'image dans le miroir est pleine et indivisible, mais pleine de vide, mais malmenée ». Cela relève d'une impossibilité fondamentale, car il n'y a pas eu de perte, elle ne s'est pas signifiée, la masse corporelle est restée sans définitions orales, sans mots pour signifier les manques, pour marquer les places vides, les jeux entre présence et absence ${ }^{10}$. C'est un corps de besoins, un corps à maîtriser, ce n'est pas un corps imagé. Besoin de rechercher éperdument des sensations fortes, de plus en plus fortes. L'évolution de «l'addicté » se fera de plus en plus vers ce biais-là. C'est un corps sans distances entre ses sensations et ses besoins de satisfaction. Sans distanciation dans la façon de le parler. C'est un corps pris au piège de lui-même. Dans l'impossibilité de se voir autonome, de se vivre autonome, il est toujours, aimanté à ce circuit infernal, rattaché à un fil, mais c'est souvent le fil du rasoir : un produit (ou son absence) et tous les gestes qui vont l'amener vers ce produit. "My kingdom for a horse " hurlait Richard III ${ }^{11}$, ce n'était qu'un royaume. Ma vie pour ce produit, dit le toxicomane, ma vie pour (ce) rien, dit l'anorexique.

Pas de manque car pas de perte. Mais de quelle perte s'agit-il? Quelle perte n'est pas réitérée. En effet, lors du sevrage premier, les protagonistes sont au moins deux à devoir perdre quelque chose, la mère et son nourrisson. Le sevrage permet une séparation après l'aliénation à l'autre, la dépendance aux soins donnés par l'autre, « la mère accompagne l'enfant dans son propre deuil. N'y a-t-il pas dans cette trajectoire [du sevrage] commune à l'enfant et à la mère, une expérience de deuil partagée? Pour que celui-ci advienne, ne faut-il pas que la mère ait eu le temps "le temps psychique" d'entendre que le sein donné est cette part détachée d'elle et offerte à l'enfant?» ${ }^{12} \mathrm{Si}$ elle se positionne comme pouvant perdre cette part de soi, comme n'étant pas elle-même complète ou complétée par l'enfant, morceau de chair la consolant, si un père est là pour aider à la séparation, elle permet à l'enfant la transition vers autre chose, un autre usage qu'alimentaire de la zone buccale, par exemple. Sinon «l'addicté » tente de ressusciter par la déchéance et la mise à mal de son corps (jusqu'à parfois l'assujettir à la perversion d'un autre) une coupure ou une perte qui n'a pas eu lieu. La perte doit être nommée pour que l'enfant puisse créer autre chose, un autre espace, un autre espoir, sinon, une part reste "non reconnue " comme altérité corporelle, comme un autre corps, un autre individu, un autre part de lui reste perdue dans un no man's land de brouillage.

D'où cette dépendance à un produit masquant peut-être la dépendance à un autre. L'impossible perte chez la mère, l'absence de réponse privative, ne permettent pas au nourrisson d'aller vers de nouvelles possessions, vers des trouvailles structurantes, d'aller vers ce qui serait bien venu de manquer. "L'addicté » n'est pas allé jusque-là, mais est resté fixé au besoin, à quelque chose de marqué dans le corps, à travers le 
corps, une sensation forte sans cesse à retrouver, sans cesse à retranscrire sur le corps dans une monotonie sans fin. Une sensation d'autant plus forte qu'aucune parole ne l'accompagne, ne la signifie, ne la transfigure.

Ce qui le met aussi face à un impossible renoncement au produit, dans une intolérance quasi excessive à la frustration. Le vide est impensable, la solitude inimaginable, impossibles à surmonter sans le recours à la stupéfiance des produits ou à leur absence, à la privation pour l'anorexique. Jamais, ils ne pourront créer le vide pour l'apprivoiser!

Le corps réclame, si rien ne vient le soulager, c'est un corps mort, un corps qui se définit comme mort, un corps tombé, blessé, inutile. Aucune négociation n'est possible, n'est pensable, aucune séparation ne peut se transcrire. Elle n'a pas pu avoir lieu, la médiation fut impossible. La confrontation au vide est inimaginable (sens plein). Il faut l'ignorer, passer par-dessus, aller droit au but vers le produit sans compromission, sans négociation, foncer et répéter sans cesse la cérémonie des retrouvailles car l'image s'est évanouie, elle s'est déchirée, le remplacement imaginaire est évité.

Des séparations sont impossibles. Des sevrages mal négociés fragilisent la confrontation aux objets diffus. Les états de dépendance au corps maternel s'instaurent sous le regard ébahi des pères trébuchant à leurs tâches. Fragiles eux-mêmes, ils n'entendent pas l'appel de l'enfant, fascinés, sidérés devant les images, nombreuses, des "vierges à l'enfant ", pourquoi y toucheraient-ils? Mais où se perd le regard de ces vierges, que regardent-elles?

21 Le monde des objets se fait de plus en plus envahissant, plus exigeant, la demande s'accroît, la réponse est de plus en plus immédiate sans distance sans paroles vraies pour la circonscrire, sans paroles réconciliatrices. Des fils lient les corps, tissent les trames de leur dépendances et les enferment, carcans lourds de conséquences néfastes. Ainsi ces «paradis que l'on dit d'artifices » ne sont ni bacchanales joyeuses et délurées, ni nirvana voluptueux et reposant sauf à être un étouffoir du désir.

\section{NOTES}

1.. J.-P. PEDinelli, P. BeRTAGNE, C. Mille, les définissent de cette façon : « La recherche avide d'un objet, la répétition, l'apparente dépendance, l'utilisation risquée du corps, la recherche de satisfaction immédiate, la proximité de la mort ou de la destruction... » in L'Information psychiatrique (vol. 63, $\mathrm{n}^{\circ} 1$ janv. 1987) ; Les pathologies addictives et leur modèle - l'incorporation -, p. 29.

2.. Cl. ESCANDE, Approche métapsychologique de la passion des drogues dans la clinique du toxicomane, thèse de doctorat, ULP de Strasbourg, janvier 1997. Livre à paraître en novembre 2002.

3.. L. COHEN, The Stranger Song.

4.. MOLIÈRE, Le Tartuffe.

5.. Nous appellerons ainsi les protagonistes de ces affections dites addictives.

6.. Au sens de Derrida. 
7.. J. R. FREYMANN, Les Parures de l'oralité, Paris, Springer-Verlag France, coll.

« Hypothèses », 1992.

8.. Thierry VINCENT, L'Anorexie, Paris, Odile Jacob, 2000.

9.. Cf. la définition des addictions.

10.. Cf. chez l'enfant le jeu du « coucou, le voilà », se cacher, se montrer, équivalent pour Freud au jeu du « fort-da ».

11.. SHAKESPEARE, Richard III.

12.. Jacques HASSOUN, La Cruauté mélancolique, Paris, Aubier, p. 41.

\section{RÉSUMÉS}

Les personnes " dépendantes » ont un rapport particulier à leur corps. "L'usage » qu'ils en font et la représentation qu'ils en ont, semblent marqués par un vide impossible à nommer. Cette problématique nous rappelle que toute séparation se négocie peut-être à l'aune d'une perte (castration orale) difficile à avaler !

"Dependent" persons have a specific attitude towards their own body. The "use" they have of it and the way they look at it seems to be characterised by an emptiness for which there is no name. This topic reminds us that any sort of separation has somehow to be negotiated with some kind of loss (oral castration) quite hard to swallow! 